\title{
IDENTIDADES MASCULINAS EN TRANSICIÓN. ANÁLISIS DE EXPERIENCIAS DE JÓVENES NILAHUINOS EN EL CAMPO Y EN LA CIUDAD EN CHILE
}

\author{
Male Identities in Transition. Analysis of Experiences of Nilahue's Young People \\ in the Countryside and in the City in Chile
} CAROLINA GONZÁLEZ* Y MARÍA CONSUELO LASO**

Fecha de recepción: 10 de marzo de 2017 - Fecha de aprobación: 21 de abril de 2017

\section{Resumen}

Los cambios en lo laboral que modificaron la propiedad de las tierras y la condición de los trabajadores de permanentes a temporales, las reformas educativas, la apertura del mundo rural a un mundo globalizado, los procesos modernizadores y la estimulación de la economía, incentivados por políticas estatales sucedidas desde los años 70, fueron procesos que transformaron los roles de productividad y las relaciones de género que venían reproduciéndose en el marco "patriarcal" tradicional de las haciendas. Estas modificaciones se expresan también en las identidades de la juventud rural y son las que el presente artículo expone, entrelazando los procesos de construcción de identidad rural y la de género, con las transformaciones estructurales, sociales y culturales, con el fin de conocer y comprender los cambios y continuidades identitarias de la masculinidad, específicamente en hombres jóvenes residentes en áreas rurales de la localidad de Nilahue, Valle de Colchagua, en la VI región, Chile.

Palabras clave: Juventud, identidad, rural, género

\section{Abstract}

The working changes in land ownership and the status of workers from permanent to seasonal, the education reforms, the opening-up of the rural world to a wide globalized world and other major changes, were encouraged by public policies oriented to stimulate the economy and modernization from the 1970s onwards. All above mentioned transformed the roles of productivity and gender relations developed within the traditional "patriarchal" framework of the haciendas. This article aims to address these modifications as an expression of the identities in rural youth, analyzing both the processes of construction of rural identity and gender, along with the structural, social and cultural transformations, in order to gain knowledge and understanding of changes and identity continuities of masculinity, specifically in young people living in the rural areas of Nilahue, Colchagua Valley, in the VI region, Chile.

Keywords: Youth, identity, rural studies, gender

\footnotetext{
* Antropóloga Social, Universidad Academia de Humanismo Cristiano. Magister de Estudios de Género y Cultura, Mención Ciencias Sociales, Universidad de Chile. Investigadora y académica Universidad Alberto Hurtado. Facultad de Educación, Centro de Investigación y Desarrollo de la Educación (CIDE), Facultad de Antropología, Santiago, Chile. Artículo enmarcado en la tesis de magister "Masculinidades Rurales: Continuidades y Transformaciones Generaciones en las Identidades de Género en la Localidad de Nilahue" (2010). Correo-e: gonzalezcardenas.c@gmail.com

** Antropóloga, maestría en Políticas Públicas en London School of Economics, Londres. Analista Hogar de Cristo, Santiago, Chile. Correo-e: consuelolaso@gmail.com
} 


\section{Introducción}

La historia de nuestro país ha estado marcada por regímenes de autoritarismo, los cuales han influido en la consolidación del sistema de dominación paternalista, fijado en la figura masculina del patrón, como dueño y señor de las tierras, el dinero y el trabajo. Este modelo de masculinidad continuará hasta nuestros días, no solo en la cultura rural, sino como base de la cultura tradicional chilena. Este hombre "dominante" imponía su poder sobre otras masculinidades "subordinadas", es decir, los inquilinos, una "especie de siervo que pagaban sus contribuciones ya no solo en especies, sino también con trabajo" (Bengoa, 1990:15), y sobre la mano de obra adicional (parientes o afuerinos) en calidad de obligados ${ }^{1}$ (Salazar, 1989; Bengoa, 1988; Rojas, 2004; Gómez, 1990). A su vez, existía una masculinidad intermedia, en la figura de los ministros o capataces, a veces más crueles que el mismo patrón.

Los hacendados y administradores intervenían directamente en los hogares inquilinos, infringiendo la autoridad patriarcal de los trabajadores, en uno de los pocos espacios en que los hombres podían ejercer su autonomía y autoridad, lo que provocaba un control excesivo de sus esposas e hijos, profundizando las formas de dominación masculina al interior del hogar campesino (Tinsman, 2009).

La cultura masculina fraguada en la hacienda, tanto en lo laboral como en las actividades festivas tradicionales, se manifestaba tanto en el arte de dominar caballos como seducir a las mujeres, constituyéndolos como atributos inseparables de la masculinidad (Valdés, Rebolledo, Willson, 1995). La movilidad dentro del espacio público mascu- lina superaba a la femenina, al asistir a rodeos, cantinas, mercados, y ferias de actividades festivas o de comercialización.

Durante este periodo, los hijos e hijas de inquilinos debían obediencia a sus padres. En esta relación los hijos socializaban con ellos a partir de su experiencia laboral en el trabajo $y$ en los roles de género, y se transformaron, muchas veces en trabajadores obligados, entregando trabajo sin remuneración, lo que no les permitía ir a la escuela más de dos o tres años. La única posibilidad que encontraban para abrir sus espacios de interacción con otros era el servicio militar, lo que además les permitía una temporal movilidad territorial aunque también en condición de subordinación, o bien, liberándose de pagar "obligación" al ser expulsados del sistema, por falta de tierras o trabajo, lo que los condicionaba a emigrar (Rebolledo, 2013).

El período previo a la reforma Agraria, significó que poco a poco se comenzara a abrir el mundo hacendal, consecuencia del accionar de grupos de iglesia, partidos políticos de izquierda y a la experiencia adquirida en el trabajo sindical de los hijos que migraron (Ídem).

No obstante, en este proceso existió una desigualdad que profundizó algunas diferencias, aumentando el poder de los hombres, por sobre las mujeres. Fueron los hombres los receptores directos de la tierra, eran ellos quienes constituían el grueso de los sindicatos rurales, y ellos fueron los definidos como actores principales en este proceso de reforma del mundo rural. Esta orientación de la reforma Agraria hacia el sector inquilino masculino, dejó fuera de sus beneficios más significativos a la fuerza de trabajo temporal (peones, afuerinos, mujeres y niños) los que fueron considerados 
como fuerza de trabajo marginal o ayuda familiar no remunerada. Quienes fueron beneficiados por las tierras reformadas, al cambiar la relación directa con el patrón por la atención preferencial por organismos estatales, agudizan sus conductas machistas, especialmente por la interacción entre hombres en el marco de las nuevas organizaciones (Rebolledo, 1997; Tinsman, 2009).

Sin embargo, las mujeres no fueron pasivas ni excluidas. Pues, si bien la reforma Agraria reforzaba algunas formas de dominación masculina (rol masculino de proveedor) erosionaba otras (el sentido del derecho sobre el cuerpo de las mujeres rurales), permitiendo que las mujeres vivieran y negociaran al interior del patriarcado, $y$, aunque éste permaneció, sufrió cambios significativos, especialmente para las mujeres (Tinsman, 2009).

En este periodo, las generaciones jóvenes ampliaron su escolaridad en cuanto a los años de educación obligatorios (se llegó a 8), así como se logró una mejor cobertura escolar por la construcción de nuevas escuelas. Esto permitió que los hijos e hijas lograran superar la escolaridad de sus padres. A ello se le sumó la consideración de nuevos horizontes, donde por primera vez tenían la posibilidad de compartir con otros niños y niñas de diferentes regiones, a través de las colonias vacacionales impulsadas por el gobierno de Allende. Además, la presencia de las mujeres adultas en el hogar, y la mencionada reducción de sus responsabilidades productivas, tuvo como consecuencia que las hijas lograran estudiar (Rebolledo, 2013).

La irrupción de la dictadura militar en septiembre de 1973, agudizó la proletarización, transformando nuevamente la tenencia de tierras, pues, donde antes existieron haciendas y donde los parceleros beneficiados de la reforma no retuvieron las tierras, aparecen propietarios con mentalidad empresarial, invirtiendo en sus explotaciones e incorporando nuevas tecnologías, reorientando la producción hacia la exportación (Rebolledo, 1997). La presencia estatal cada vez más débil en el campo chileno, disminuyó y restringió las posibilidades de reproducción de los parceleros de la reforma agraria y de los campesinos lo que también influyó en que las formas de trabajo se modificaran.

El proceso de globalización y los cambios en la estrategia productiva con la internacionalización de los proceso productivos, en el marco de instauración del neoliberalismo, contribuyeron a una gradual incorporación masiva de la mujer al mercado de trabajo temporal, desarrollando labores de tipo manual, especialmente en relación a la manipulación y embalaje de la fruta, mientras los trabajos rurales masculinos permanentes tienden a desaparecer. Esto trajo cambios en la estructura de la vida privada del mundo campesino, como también en el ordenamiento de los territorios donde se asentaron originalmente los trabajadores, formando caseríos y villorrios alrededor de los centros de trabajo, así como nuevos centros rurales y urbanos. Esto se produce sobre la base de dos procesos: la expulsión de las fuerza de trabajo por las distintas formas de tenencia de la tierra hasta los años setenta (fundos) y aparición de una nueva forma de producción (Valdés, 1998; Rebolledo, 1997; Salazar, 1989; Bengoa, 1990).

Estos cambios de vida de los habitantes rurales a través de los procesos de campesinización y descampesinización, de urbanización del campo y aparición de nuevas formas de ruralidad, del 
cambio de residencia, del trabajo de la mujer, y la ruptura en las relaciones comunitarias y de vecinos (Rebolledo, 1997; Valdés, 1998, Salazar, 1989) provocan cambios en la provisión masculina al hogar, transformándose en una actividad compartida con la mujer, puesto que ella también contribuye con un salario, porque si bien, en el pasado también trabajaba, esto se daba en el marco de la hacienda donde el hombre era el proveedor mayor. A su vez, comienzan a emerger y desarrollarse con fuerza las identidades juveniles rurales, algo que en los estudios de la historia del agro ha sido difícil de encontrar, ya que por lo general, los niños tenían su paso a la adultez, en ritos socioculturales asociado a las relaciones con sus pares adultos, en ambientes de distinción, y especialmente de trabajo.

Es por ello que en este artículo abordaremos tres elementos que nos parecen claves para comprender los cambios ocurridos en lo que conforma la ruralidad en Chile hoy en día, la combinación entre la juventud y masculinidad, como producto de las transformaciones en las relaciones de género al interior de los hogares, y la identidad juvenil rural como una secuencia de cambios históricos y políticos que hacen que la frontera entre lo urbano y lo rural sea para este segmento más flexible y permeable que para otras generaciones.

Para este artículo, se analizó parte de los resultados de una investigación realizada en el marco del Proyecto de FONDECYT "Cambios en las identidades y relaciones de género en el ámbito rural de Chile Central, 1940-2010" bajo la responsabilidad de Ximena Valdés y de Loreto Rebolledo como co-investigadora y tutora de la Tesis de Magíster en Estudios de Género y Cultura de Carolina González.
El trabajo en terreno se llevó a cabo durante el año 2012, en la localidad de Nilahue, comuna de Pumanque, Provincia de Colchagua, en la Región del Libertador General Bernardo O'Higgins, específicamente en la Localidad de Nilahue Cornejo. Los entrevistados fueron jóvenes descendientes de padres o abuelos que vivieron al interior de fundos.

Este estudio fue de tipo cualitativo basado en entrevistas que se realizaron en varias visitas a terreno que incluyó la observación y participación en distintos espacios mixtos y masculinos. Asimismo, para la recolección de información, se utilizaron las entrevistas en profundidad e individuales a diez jóvenes considerando la experiencia educativa prolongada (educación básica y media, y superior), relativamente ausente en las otras generaciones mayores. Además se realizó entrevistas grupales a partir de conversaciones semi-estructuradas, donde se buscó comparar opiniones contrarias, similares recuerdos, percepciones y opiniones sobre modelos de comportamiento identitarios, sobre modos de hacer y vivir, según su pertenencia y género. Estas entrevistas se desarrollaron en dos grupos, uno de tres hombres, y otro mixto, compuesto de 5 hombres y 5 mujeres. Esto nos permitió además diferenciar las representaciones, características e identificaciones propias y externas de las experiencias y consideraciones personales sobre su identidad desde la dimensión discursiva.

\section{Identidades en juventud, género y ruralidad}

La literatura en relación a jóvenes y ruralidad es incipiente, y siendo los estudios en mayor medida orientados a la juventud urbana, por 
lo que cabe preguntarse en qué medida se parecen a los jóvenes rurales, especialmente para el diseño de políticas públicas para este sector. La juventud como concepto nace de la burguesía capitalista del siglo XIX, donde los padres con mayor solvencia económica pudieron mantener a sus hijos retrasando así la edad en que éstos debían comenzar a trabajar (Silva, 2002 en Barrera y Gormaz, 2009). No obstante la juventud se asocia más con las culturas juveniles, tribus urbanas y otros aspectos relacionados con la búsqueda de la identidad propia de esta fase del ciclo vital.

Las definiciones sobre la condición juvenil plantean tensiones importantes para distinguir al sujeto joven rural. En algunos casos ${ }^{2}$ se apela a determinantes económicos y sociales, es decir, un sujeto que asume funciones adultas rápidamente debido a sus regímenes de matrimonios más tempranos, ausencia de periodos formativos extensos, a una inserción laboral temprana, un periodo de moratoria ausente o disminuido, que finalmente responde a un sujeto campesino de menor edad (o no adulto en función de adulto) pero que no alcanza a formar un cuerpo social con identidad. Frente a esto, Durston (1998) menciona que este concepto de juventud (fenómeno reciente y urbano), sería una visión parcial e insuficiente de la compleja y diversa realidad juvenil, que alimenta un estereotipo sobre las etapas de vida en el campo, exponiendo el desconocimiento de sus realidades concretas. De esta manera, se establece la complejidad de que, si un joven rural, trabaja, es jefe de hogar, casado y con hijos, deja de ser joven, y pasa a la categoría adulto, provocando el cuestionamiento de esta categoría en este tipo de contextos.
Bajo esta perspectiva, es importante entender las diferencias que aparecen en la construcción de las identidades juveniles, estableciendo espacios de interrelaciones de las áreas urbanas y rurales, con sus respectivas tensiones, evitando el encierro conceptual frente a lo contextual. Frente a esto, el investigador, Yanko González Cangas (2003) nos expone cómo las primeras experiencias identitarias juveniles deslocalizadas han sucedido en nuestro país, en distintos periodos de tiempo:

\footnotetext{
"Aparecen en las décadas del treinta al setenta, con los abundantes enrolamientos al Servicio Militar por parte de los jóvenes y la alta ocupación en el servicio doméstico urbano por parte de las mujeres jóvenes. Sin embargo, en las décadas posteriores (ochenta y noventa) estas experiencias se radicalizan con la neoliberalización del agro, la modernización del transporte y las comunicaciones y la expansión educativa, que hacen a las nuevas generaciones acceder a la enseñanza secundaria, técnico-profesional o al trabajo fuera de la comunidad en pesqueras y buques factorías internacionales. La mayoría son experiencias de deslocalización estacional que implican el regreso e impresionan a la comunidad de origen con el fermento de un imaginario juvenil” (González, 2003: 171).
}

Considerando lo anterior, nos parece fundamental reflexionar sobre la diferencia en la constitución del mundo juvenil rural en comparación con el joven urbano, en la medida en que los fenómenos de aplazamiento o moratoria, socialización, elementos identitarios, entre otros, adquieren significados distintos en los jóvenes del agro, pero tienen igual ocurrencia. En décadas anteriores se podría argumentar que el periodo de moratoria de los jóvenes no siempre tenía características formativas, en el cual la escuela tenía menos importancia como agente socializador, en comparación a los amigos, la familia y los medios de comunicación, además de la característica incorpo- 
ración temprana a las labores productivas (Ídem.). Para el año 2000 los jóvenes rurales aún se diferenciaban de sus pares urbanos, al provenir de sectores de pequeños agricultores o de familias con terrenos cultivables, $y$ al ser obligados a ingresar tempranamente a las labores agrícolas o asociadas, conservando a su vez, su dependencia familiar, que continuaba desarrollándose en un prolongado tiempo (Zapata, 2000). En este aspecto, es importante mencionar que, actualmente, en el caso de los periodos de prolongación de la dependencia, ésta ha tomado mayor relevancia como consecuencia de las nuevas trayectorias educativas y laborales de los jóvenes, sumado a la alta migración temporal, el contacto urbano y la globalización mediática, además de la importancia que adquieren en la socialización y consolidación de la juventud, los pares y los medios de comunicación, a la par que las escuelas, liceos, internados, universidades o centros de formación técnica.

Así, como sostiene Feixa (1999), ante la pregunta de si existen culturas juveniles en el mundo de la pequeña agricultura, debemos entender que si bien las culturas juveniles se asocian a las ciudades, su difusión va más allá de las divisiones rural/urbano, y que más allá de comprobar existencia, se debe buscar y analizar los contenidos de aquellas probables culturas juveniles.

En este sentido, la juventud, entendida desde perspectiva antropológica, como una construcción sociocultural relativa en el tiempo y en el espacio, si bien tiene una base biológica, debe ser entendida desde un contexto histórico y sociocultural (Feixa, 1998), cobra relevancia para entender el contexto actual, puesto que, como consecuencia de los procesos que hemos mencionado "la generación juvenil actual posee una familiaridad con la cultura y valores de la modernidad mucho mayor que la generación de sus padres (así como una mayor educación formal), y a la vez mantiene un arraigo con la cultura local" (Duhart, 2004:123), lo que los convierte en un actor "híbrido" al moverse entre distintas culturas, brindando características para la búsqueda de un modelo de desarrollo que el medio ambiente y las identidades culturales locales (Ibíd.), transformándolos en actores determinantes para el desarrollo de las zonas rurales en general, proponiendo también nuevas propuestas y definiciones sobre lo rural.

Con el conocimiento internalizado y naturalizado de los cambios en los derechos laborales, la declaración de los derechos de la infancia, y los derechos de la mujer, las transformaciones en el contexto educacional ${ }^{3}$, sumado a los procesos de modernización que afectan las comunicaciones, la información, el desplazamiento, y las influencias externas, se construye su identidad de manera más urbana, distante y crítica de lo tradicional y local, pero que conserva ciertos elementos tradicionales. Estos antecedentes configuran en parte lo que se entiende como nueva ruralidad, que promueve una reinterpretación de la ruralidad como algo más bien incierto e inestable en contraste con una cultura imperativa e inmutable, más cercana a una transición que una dicotomía, una frontera permeable donde los jóvenes son clave para comprender los flujos que atraviesan dicha frontera.

La entrada de internet, el manejo de los dispositivos electrónicos y plataformas digitales ha sido incorporado desde hace años no solo por las instituciones del estado a través de la educación obligatoria (Programa Enlaces 
por ejemplo) sino que han sido utilizados por jóvenes y niños quienes transmiten este conocimiento a personas más adultas. De tal manera que su juventud e identidad juvenil se expresa no solo a través de la ruralidad sino que más bien en aspectos transicionales complementarios, atendiendo las características permeables y transformadoras de la identidad.

Junto con lo anterior, nos parece importante comprender que la identidad, al ser una categoría relacional que se va configurando durante toda la vida del individuo, en condiciones socio-históricas particulares y a partir de la participación del sujeto en los procesos de producción y reproducción social, se incorporan puntos de referencia del orden social (Reyes, 2009; Rocha, 2009). En este sentido, la identidad de género, entendida como un constructo sociocultural en base a la diferencia sexual (Lamas, 2002), cuya dimensión política significa plantear el género como fuente de desigualdad, es decir, como una forma primaria de relaciones de poder (Viveros, 2000), es una dinámica que se construye en la interacción social y la experiencia individual, inherentemente históricas, cuya construcción y transformaciones son parte de un proceso político, a través de un individuo-agente constructor social y culturalmente inscrito, que influye en el equilibrio de los intereses sociales y por ende, en el curso que tomen los procesos de cambio social (Valdés \& Olavarría, 1997; Viveros, Olavarría \& Fuller, 2001).

En este contexto, lo masculino condensa, además del conjunto de rasgos característicos de quienes nacen con ciertos atributos anatómicos, cualidades humanas (actividad, la razón, el poder, la fuerza, por ejemplo) construidas en la oposición binaria por la cual lo femenino se convierte en ausencia y negación (Fuller, 2001). Esto trae como consecuencia que hombres y mujeres se desarrollen en dos mundos diferentes: La mujer en lo doméstico (lo privado), reproducción, cuidado y educación, y el hombre en el resto del mundo, en la producción, creación y lo político (lo público) (Badinter, 1993). Bajo esta idea, se comprende cómo los jóvenes hombres buscan responder al imaginario social construido a partir de un discurso del ser hombre (Olavarría, 2001), el cual observan y viven en sus relaciones cotidianas, en escenarios de encuentro juvenil (Romero, 2006).

\section{Nilahue: Características de la población}

De acuerdo a los datos del Plan de Desarrollo Comunal PLADECO de la I. Municipalidad de Pumanque ${ }^{4}$ 2014-2017, la población de la comuna se reconoce como $100 \%$ rural, aunque parte importante de ella viva en zonas urbanas, concentrándose en Pumanque y Nilahue Cornejo. La evolución de la población de la comuna ha ido decreciendo en los últimos años, presentando una variación de $-10 \%$ desde el Censo de 2002 al 2012, donde la población estimada fue de 3.442 y 3.095 respectivamente.

Asimismo, según datos entregados por la municipalidad, el grupo etáreo con mayor cantidad de personas es el rango entre 45 a 64 años (909 personas) representando un $29 \%$ de la población total de la comuna. A este tramo le siguen aquellas personas entre 30 y 44 años con 593 habitantes (19\%) mientras que la población joven está representado por personas entre 0 y 14 y 15 y 29 años con un $18 \%$ y $19 \%$ respectivamente. 
Estos antecedentes son importantes para comprender que estamos en un escenario donde el cambio en la estructura económica y social de la zona está afectando la cantidad de personas residentes en localidades rurales, principalmente en población más joven.

La pérdida de las fuentes de trabajo del mundo campesino, tal como lo conocieron los padres y los abuelos, produjo un desplazamiento veloz de población hacia centros con mejor oferta laboral, lo que se ve acentuado con nuevas formas de inclusión de los jóvenes a la educación superior, emigrando muchos de ellos a centros urbanos en busca de oportunidades de estudio y trabajo.

\section{La identidad rural de los jóvenes en época de modernización y globalización}

La herencia hacendal, la oportunidad de trabajar la tierra como medio de subsistencia de la familia y de ser propietario de la tierra que se trabaja a partir de la reforma, la pérdida de terrenos, las opciones de trabajo que entregan las nuevas condiciones económicas actuales, todo esto unido al mantenimiento y reproducción de los patrones de masculinidad tradicionales ligados al mundo rural, forman una virilidad cuyas principales características son la tierra, el trabajo y la producción, el rol de proveer, la lealtad y la responsabilidad. Esto se observa tanto en las descripciones que hacen de sí mismos como en las que hacen de los hombres citadinos.

Frente a la masculinidad opuesta externa, los jóvenes criados en un ambiente rural también buscan o identifican una diferencia en la constitución del ser hombre de campo frente a un hombre de ciudad, considerando ciertos valores, el conocimiento de la tierra e incluso cierta ideología.

"Mis amigos son de ciudades. Ellos son un poco más locos. Tienen otra ideología" (Hombre, 24 años).

"Yo creo que también va el tema valórico de la gente del campo. Porque siempre cuando la gente del campo va a la ciudad se toma como gente trabajadora, por gente honrada" (Hombre, 18 años).

De esta manera, aparece el campo como lugar idílico, donde se conjuga la naturaleza, la tranquilidad del vivir, los valores tradicionalmente cristianos, todo esto en un espacio donde se ha desarrollado históricamente masculinidad tradicional. Sin embargo, los cambios contextuales en la última parte del siglo $\mathrm{XX}$, vividos en los más jóvenes, expresados en el ingreso y la oportunidad de asistir a una escuela en un periodo de tiempo más amplio y constante, además de los contactos en un mundo globalizado, sumado a la llegada de la adolescencia y la juventud como etapa etaria, han producido que los procesos de identificación y configuración de género en esta generación se acerquen más a la idea de jóvenes urbanos, especialmente a través del consumo y expresión de nuevas músicas, nuevas costumbres, vestimentas e identificaciones (tribus urbanas) y al cuestionamiento de la vida en el campo.

"Es que igual la vida del campo es sacrificada. O sea, al menos para mí, que estoy estudiando, no tengo manos para el campo. Es que ahora uno tiene que estudiar, porque acá en el campo no hay trabajo. $Y$ es muy sacrificado. Se trabaja mucho y se gana poco. (...) No soy anti campo, si igual me gusta el campo. Pero no para venir a trabajar. O sea, igual trabajaría en el campo, pero es difícil por lo que estoy estudiando. Eso es lo complicado. Me gustaría, pero es difícil. La otra vez trabajamos en el campo, instalando... pero por dos o tres semanas, más no, y terminé con las manos 
reventadas. No soy como mi tío, que tiene las manos bravas. Pero entre campo y ciudad, el campo me gusta más" (Hombre, 24 años).

"Me gusta el campo. Es filete. Más que la ciudad. Es mucho mejor. Pero no me siento tanto un hombre de campo" (Hombre, 17 años).

Para ellos, los proyectos de vida y de familia se enfocan en salir de la localidad rural, pero a lugares donde se repitan parámetros del campo (belleza y tranquilidad) incluso dentro de Santiago, o bien, dejando estos terrenos para visitas vacacionales o de retiro. Bajo estos parámetros, la idealización local se conjuga con nuevas herramientas y anhelos modernos.

En busca de la tradición

Frente a la idealización del espacio rural, emerge la responsabilidad que tienen de mantener la cultura rural, y frente a la idea de que es posible que en ellos la distinción cultura urbana/cultura rural sea más débil y difusa, los jóvenes tienen una opinión bastante clara de lo que implica transformar aquellas costumbres, dada las actuales condiciones que sus padres o abuelos no tuvieron, como el contacto exterior con el mundo globalizado, la superación de ciertas diferencias que se relacionan con un nivel social menor, entre otros. En esta investigación observamos cómo continúan presentes ciertas costumbres que los jóvenes aprendieron desde pequeños, que les permiten sentir parte de su identidad de campo, y que incluso, lo practican con orgullo. Esta contradicción se entiende en un mundo donde quieren mantener el origen y las costumbres masculinas del campo y la distinción con el "otro" de la ciudad, pero a la vez se presenta con "no ser menos" o tan diferente entre sus pares, o bien, como un paso lógico al conocimiento que se tiene de nuevos estilos y formas de expresión que quizás los identifique más como jóvenes y no tanto como hombre de campo. En consecuencia, la influencia de los estilos musicales y tribus urbanas han disminuido las preferencias por el canto a la divino ${ }^{5}$, usando en algunos casos, al hip-hop como una forma de expresión musical, en la cual se utiliza el mismo patrón de improvisación -a veces- o de repetir y memorizar, aunque las temáticas son críticas sociales globales, más que de costumbres o historias locales. No obstante, también existe la conciencia de una identidad rural que se resiste a desaparecer, pero sí es capaz de transformarse, y donde ellos, son un factor fundamental.

\footnotetext{
"Yo dejé de cantar canto a lo divino en primero medio. Es que uno cambia, cuando uno ve a la gente de afuera uno cambia. Hasta ese momento yo vivía solamente en este mundo. Después uno conoce otras cosas. Pero no me dejó de gustar. No he dejado de practicar cosas que se hacen en el campo" (Hombre, 17 años).
}

Aspectos tradicionales: El caso del rodeo

En este espacio de competencia y dominación de la naturaleza es donde se observan los atributos masculinos rurales por excelencia: Control y conocimiento de las bestias, fuerza, exhibición y búsqueda de reconocimiento. En ellos se expresa el deseo de mostrar su capacidad para domar y de triunfar, y a la vez, se aprecia la importancia de la aprobación "homosocial" y la admiración de sus congéneres y de las mujeres.

El varón que no participa de esta instancia, no solo arriesga la búsqueda y posible reconocimiento de su valentía y virilidad, sino también desafía la identidad del hombre de campo, y 
su trascendencia en el tiempo, como un valor esencial en la conformación masculina de esa localidad. El uso del caballo, el poncho y el sombrero eran costumbres que en estas instancias adquirían un aspecto de ritual y diferenciador de status y de género, donde el alcohol cobraba un importante papel en el desenvolvimiento social, y donde las mujeres eran confinadas a las tareas domésticas y al servicio de los varones y la fiesta. Esta tradición es realizada en la actualidad por varones jóvenes que quieren mantener viva esta tradición importante en su localidad.

"De hecho corro en rodeo. No he dejado de practicar cosas que se hacen en el campo" (Hombre, 17 años).

En el doble dominio y control, sobre el caballo y el bovino, se reflejan la necesidad de adquirir mando, jerarquía y poder. El estar sobre el caballo lo posiciona, no solo como un varón, sino como un varón de campo, como sus antepasados lo hicieron, y en los cuales se conformó "un estilo masculino en que el dominio del caballo, la presencia en faenas y ritos festivos contribuyeron a establecer mecanismos de integración social particulares" (Valdés, 2000).

La figura del hombre a caballo, con una postura y una actitud viril y fuerte, con destreza en el dominio de la naturaleza y el control sobre las mujeres, es parte del ideal masculino de huaso, que encontraba en el pasado su espacio de expresión en una actividad ganadera productiva, pero que actualmente, con los cambios en la vida rural y la modernización, es parte de una revalorización y resignificación de este tipo de instancias rituales del mundo campesino y de su imagen varonil, adecuadas en el contexto festivo y no como parte de la faena laboriosa.
El trabajo como rol masculino

y el paso a la adultez

En todos los casos en que nos acercamos a las historias de hombres rurales, uno de los factores que parecía inmutable en el tiempo, es el del trabajo masculino desde la infancia, el cual se presenta y desarrolla como un rito de tránsito de niño a hombre, de conocimiento, de responsabilidad e identidad, como un elemento simbólico que consolida y valida su ser social varonil, en su rol de proveedor y productor, resultado de una preparación masculina de conocimientos heredados de su padre, y la responsabilidad de saber que el trabajo no puede faltar ni detenerse nunca, si quiere poseer una de las características básicas de la hombría rural. El trabajo se conserva como un factor de rito de paso.

Para las generaciones mayores, el trabajo responde a la necesidad y obligación, sobre todo si se es el primogénito, mientras que, en los jóvenes, si bien comienzan a trabajar desde pequeños, el valor y el sentimiento sobre el trabajo varía, pues para ellos, al no ser siempre una necesidad económica imperiosa, pasa a ser una carga simbólica dentro del proceso de configuración de identidad masculina y de integración social. Esto no quiere decir que sus ingresos no sean una fuente importante de contribución para la familia, para sus ahorros futuros o para sus propios gastos.

\footnotetext{
"Yo desde chico, no me acuerdo en realidad. Pero más o menos siete años, en el fundo de un tío acá, haciendo etiquetas, cuestiones así, nada, así como el packing. Trabajábamos donde mismo. En el fundo donde mis tíos, pero ellos son de Rancagua. La Familia te lleva. Me llevaban mis primos, mis tíos, decían que fuera a trabajar allá. A mí por lo menos no me llegaba la plata porque era familia. A mi igual me pasan, pero de repente" (Hombre, 16 años).
} 
"Pero es que igual va en una cosa de costumbre porque igual uno empieza desde chico a trabajar. Entonces después es normal trabajar para uno" (Hombre, 17 años).

En este caso, la importancia del trabajo influye en que los jóvenes se asuman más grandes, pero no adultos, lo que lleva a un factor de diferenciación de identidad local con otros jóvenes, adquiriendo características de hombre/adulto, pero no en el hecho de demarcar una identidad adulta tan clara como en otras generaciones, debido a la concepción de juventud prolongada presente en la actualidad.

También se refleja la concepción que se tiene del trabajo en el campo, en la tierra, pues los jóvenes lo comprenden como una acción ardua y sacrificada, incluso si no se participa permanentemente de él. Estos varones expresan otras opciones o aspiraciones para desarrollarse laboralmente, contando con la posibilidad de elegir salir de su pueblo o permanecer en él. Muchos de ellos plantean la idea de regresar una vez consagrados, o bien regresar solo como una visita, pues su futuro y sus posibilidades están en la ciudad, no en su localidad.

"Yo, quiero entrar a la escuela de Investigaciones. Pero en el campo no. Es que yo tuve una mala experiencia que cuando llegue aquí, llegamos sin ni un peso, porque llegué con mi mamá, después que se separaron. Y mi mamá trabajaba en el campo, y no me gustaba ver como mi mamá trabajaba en el campo. Entonces yo quiero surgir" (Hombre, 17 años).

De este modo, los varones jóvenes nos muestran otra manera de marcar diferencia con sus pares y su condición rural. La opinión que tienen los jóvenes entrevistados sobre aquellos que no optan por esa vía y se quedan en su pueblo ellos es de mediocridad, de pocas ganas de surgir.
"Pero algunos tienen una mente muy penca, algunos quieren cuarto medio para sacar la licencia y manejar tractores no más, o algunos van a estudiar técnico agrícola para ser jefe de fundo y cuestiones así" (Hombre, 17 años).

Para los jóvenes entrevistados, y a diferencia de sus abuelos, dejar la escuela no es una opción, pero si deben tener esta doble jornada que puede influir en el peso extra que siente la generación joven en la actividad laboral.

Si bien su discurso puede ser más urbano e informado, los jóvenes de Nilahue mantienen cierta relación con el trabajo, como un determinante del ser hombre en una cultura tradicional. Ciertamente, ninguno de ellos ha estado sin trabajo, o no ha trabajado en las labores correspondientes a los conocimientos del padre o de la figura paternal presente, no obstante, su opción por el trabajo se basa en la independencia que el esfuerzo le otorga y en las presiones que tienen de sus padres y de sus madres, que intentan hacer reales sus deseos de formar hombres que lleven consigo las características identificadas como propias del hombre de campo: esforzado, trabajador inagotable y respetable con su entorno.

En contraposición, las mujeres jóvenes no siempre trabajan. Esto nos muestra una diferencia importante en la respecto del trabajo para los hombres, frente a la experiencia laboral de las mujeres. Cabe destacar que a una joven (14 años) habiendo sido aceptada dos años antes en Los Arándanos, como temporera, este año no fue aceptada por su edad. Al parecer hay nuevas leyes que sancionan el trabajo infantil, sin embargo, otros jóvenes varones de la misma edad sí estaban trabajando de temporero para la misma fecha. Creemos que es probable que haya un sesgo de género en ese límite o se naturaliza más el hecho de que el hombre joven no tiene edad mínima para trabajar. 
En relación a la diferenciación identitaria y a las opciones de educación que poseen, el desplazamiento físico a internados o a colegios masculinos ubicados fuera de la localidad, generalmente a ciudades cercanas, implica un alejamiento de sus familias y de su localidad la mayor parte del año, pues su hospedaje permanente se desarrolla en la misma institución educativa, en posadas o piezas arrendadas. Asimismo, se demuestra las percepciones y comprensión respecto al espacio masculino en el cual están insertos, valorándolos como una etapa de crecimiento y como símbolo de responsabilidad y madurez, elementos propios de la masculinidad.

\footnotetext{
"Así uno se acostumbra antes, por ejemplo, las mismas chiquillas no están acostumbradas a estar fuera de sus familias. Algunas sí y otras no. $Y$ después cuando tengan harto tiempo va a pesar" (Hombre, 17 años).

"Aparte que nos ayuda a tomar bien las decisiones, con lo que aprendimos afuera, estando solo. Le tomas el valor a las cosas también. De repente nos empezamos a sentir más grandes" (Hombre, 17 años).
}

El comienzo de otros sueños y nuevos proyectos

Los cambios que conlleva la modernidad (carreteras, televisión, transporte, telecomunicaciones, etc.), el desplazamiento de los límites entre lo rural y lo urbano y los nuevos contactos con realidades, grupos musicales, expresiones artísticas, religiosas y músicas del mundo, rompen con lo tradicional del campo chileno, y comienzan a influir en los jóvenes con tendencias nuevas, que van modificando y resignificando ciertos elementos tradicionales, pero que también cierran espacios de diálogo y de comprensión intergeneracional.
Para los jóvenes, las formas de pensar, actuar y manifestarse son los principales elementos de confrontación, no solo entre generaciones, sino con la cultura rural que los rodea, generalmente asociada a una idealización de la relación campo-naturaleza, pero también con un estado de educación e información menor, y a un aislamiento espacial y cultural.

La música electrónica, el metal y el hip hop son las preferencias en sus gustos musicales y en sus formas de vestir, lo cual causa un gran rechazo en la población mayor y una crítica a su atuendo y a su poco rescate de sus raíces.

"Me ven vestido así, piensan que soy delincuente. Me molestan porque me visto de ancho no más (hiphopero). Habían dicho que cantara algo más típico" (Hombre, 17 años).

"A uno lo critican como se viste. Me dicen 'vos soy volado', 'soy guachiturro', es mucha ignorancia" (Hombre, 17 años).

La existencia de un rechazo a una nueva cultura, manifestada en jóvenes hip-hoperos, pero también en todos los demás jóvenes, lejanos a su tradición rural, nos revela también la falta de comprensión de un fenómeno que las generaciones mayores no alcanzaron a conocer, y que es, en esta época, donde se ve con mayor fuerza sus elementos identitarios. Hablamos de la adolescencia y la juventud. Esto, creemos, es porque en los tiempos de sus padres y abuelos, el paso de niño a adulto era inmediato, y en este cambio de identidad etaria, eran ellos, como adultos los que se sentían parte responsable de mantener su cultura identitaria rural. Esto no sucede con una juventud que busca diferenciarse y expresarse de manera distinta a como lo hacen los niños y los adultos, ya sea de su localidad como del resto de la sociedad. 
Por otro lado, para los jóvenes, el nuevo mundo se presenta conectado, con oportunidades y con información, algo que hacen suyo, como parte también de su identidad. Los jóvenes rurales se acercan cada vez a sus jóvenes pares urbanos, pero aun -conscientes o no- siguen reproduciendo ciertos elementos que los mayores no logran apreciar a simple vista, es por eso la existencia de un prejuicio de "delincuentes", de "vagos", o bien la constante crítica a sus manifestaciones.

Las nuevas identidades juveniles, asociadas a creencias y filosofías distintas a las tradicionales, les dan un punto de vista crítico frente a lo que la gran mayoría de los pobladores expresan e identifican. Nuevas religiones o incluso un ateísmo ambiguo se sumergen en rituales tradicionales bajo el marco del catolicismo, pero ahora cuestionado como institución, a diferencia de otras generaciones más conservadoras.

\begin{abstract}
Sin embargo, todos tienen un patrón de creencia y de rituales católicos, como catecismo, bautizo, misas, etc., utilizando estos rituales como una forma de participar colectivamente en ceremonias locales o como distracción, pero lejos -desde el discurso- del sentido de veneración o celebración de la virgen o del santo: "Yo tengo creencias de todas las religiones, tengo creencias de la iglesia evangélica, algunas de la iglesia católica". Asisten a las misas "porque es obligación. Te sacan de la sala y es bueno porque no tienes clases" (Jóvenes Nilahuinos).
\end{abstract}

La irrupción del mercado también va modificando los elementos utilizados en el día a día. La ropa utilizada tradicionalmente por las generaciones mayores se pierde con las nuevas identidades juveniles, que adquieren elementos extranjeros resultado de su apertura al mundo y a otros lenguajes y estilos. Sin embargo, en la generación mayor también se puede observar que ciertos elementos tradicionales en su vestimenta ya no son utilizados, remplazando el poncho, la ojota y la chupalla, por jockeys y poleras. Sus rasgos de vestimentas son compartidas por la generación intermedia y mayor, no así con los más jóvenes, que buscan en elementos más amplios para manifestar su búsqueda de identificación y validación como generación. Su desplazamiento cotidiano es a través de bicicletas, pues solo en contadas ocasiones se vio a familias desplazándose a caballo.

Los jóvenes más urbanos, tienen otros sueños

Las influencias externas han ido cambiando los sueños y las proyecciones que tienen los jóvenes, quienes, en su desarrollo identitario más urbano, aunque conservando algunos elementos de la vida de campo, van abandonando aspectos identitarios importantes, así como también van dejando la localidad que los vio nacer, lo que tiene como resultado una población juvenil disminuida durante gran parte del año.

Esta generación transcurre su vida en un sistema que los obliga a salir para buscar un mejor futuro, por las escasas oportunidades en su lugar de nacimiento. Al preguntarles a los jóvenes qué querían hacer, todos respondieron que querían estudiar una carrera (universitaria en su gran mayoría, técnica la minoría), vivir en una ciudad, con otros ritmos y oportunidades, viajar y recorrer el mundo, y darle los mejores beneficios a sus familias.

Bajo estas aspiraciones y anhelos, la vida de trabajo en la tierra se aleja de sus intenciones. El 
trabajo al sol y esforzado no es la mejor alternativa para ellos, en el futuro, debido a que el gran esfuerzo que significa trabajar con las manos y la tierra no tiene la recompensa en dinero que ellos esperan.

\section{Estereotipos, roles de género y la división sexual del trabajo}

Persistencia de ideas tradicionales en torno a lo femenino

Si bien es posible observar un cambio en las generaciones más jóvenes, esto no implica que la responsabilidad y las tareas realizadas tanto por hombres como por mujeres sean iguales, pues aún subsiste en los jóvenes, la idea de que el trabajo doméstico no es tan importante, argumentando que el hombre trabaja afuera, "bajo el sol castigador", para luego trabajar en su campo, mientras que la mujer trabaja en cosas "de menos complejidad", más cómoda, "debajito de sombra" (Hombre, 18 años). Dejando en claro -para ellos- que "es más fácil ser mujer en el campo" en lo que se refiere a las labores y al esfuerzo diario, invisibilizando otro tipo de labores tradicionalmente ligadas a lo femenino.

En sus comentarios es posible advertir una estereotipación de la mujer y de la labor del hombre, estableciendo comparaciones en la que identifican una diferencia "injusta", dado que, para ellos, el trabajo más pesado es el de hombre rural, aunque es preferido frente a la "inactividad" o "dependencia" femenina, y donde, el embarazo, sería la salida fácil para las mujeres rurales.

"Las que son dueñas de casa son las que no quieren estudiar ni hacer nada, entonces qué mejor que emba- razarse y con eso lo hacen todo" (Hombre, 15 años).

"La ventaja de ser hombre aquí es que son los que trabajan, porque las mujeres jóvenes no. Los hombres jóvenes sí. Como que las mujeres jóvenes están preocupadas de ellas" (Hombre, 16 años).

"La mujer no hace nada, cocinar, sentarse y nada más. El hombre trabaja y quita y trabaja. (...) El hombre trabajando al sol po, no como la mujer que trabaja en la casa debajito de sombra, no po, no es lo mismo" (Hombre, 18 años).

Es interesante ver como las opiniones llevan una percepción sobre el egoísmo femenino, frente al rol de cuidador y proveedor que debe tener el hombre. Esta contraposición de valores y roles determinan las maneras de relacionarse y de entender el rol de género propio y ajeno en la conformación de una relación. Además, se aprecia una desvalorización del rol doméstico, lo cual es importante señalar, puesto que son una generación que aspira a salir de eso.

Los jóvenes poseen una visión particular sobre las relaciones amorosas o "pololeo" y el compromiso con las mujeres, determinada por la imagen femenina de "control", frente al ideal masculino de "independencia", lo que los lleva a definir las relaciones formales con el género opuesto como demandantes o falto de libertades.

Frente a todas estas concepciones del sexo, la mujer es calificada como "cada vez más sueltas", evaluando a todas las mujeres como prostitutas, al apropiarse eróticamente de ellas, como parte de la cultura política patriarcal.

\footnotetext{
"¡Ahora las minas son más pelás"! Es que antes eran señoritas. Ahora tú les decís ‘¿vamos para allá?'-‘ivamos!"' (Hombre, 17 años).

"Es que ahora se empezó a pintar y ahora parece mono, empieza con "pu" y termina con "ta." Es que
} 
anda con escotes, muy... si, en realidad parece. Es que cambió caleta" (Hombre, 15 años).

Por otro lado, en lo que se refiere a la crianza familiar, es posible encontrar justificaciones frente a ciertas diferencias entre los géneros, cuyas desemejanzas, condicionaría el trato hacia ellos y ellas.

"Son muchos más protectores con las mujeres que con los hombres. Es que a las mujeres uno las pesca y les hace cualquier cuestión, una mujer a un hombre no creo" (Hombre, 18 años).

La nueva visión

en torno a las relaciones de género

Las influencias externas, las situaciones contextuales, las nuevas oportunidades, que suceden en un marco de derechos y de aperturas de temas que cuestionan y muestran otras realidades, han hecho que en localidades rurales, se cuestione y se reflexione sobre la injusticia, abuso de poder y la violencia de la crianza observada en las generaciones anteriores. Al respecto, los jóvenes tienen una postura crítica, reconociendo ciertas situaciones de violencia injustificada y de desigualdad de géneros en su crianza.

"Es diferente también el trato de los papás con los hombres y las mujeres. Con nosotros son, por lo que se ve, más brutal que con las mujeres" (Hombre, 17 años).

Frente a este reconocimiento de prácticas de relaciones paternales violentas y lejanas, emergen nuevas valoraciones, las cuales abren espacios al anhelo de una paternidad más afectiva. No obstante, aún se busca un equilibrio entre la comunicación, el respeto y el orden.
"Pero antes no había comunicación pero sí había golpes, por eso la gente se criaba "mejor", pero ahora que es lo que pasa, no hay golpe pero no hay comunicación, todavía no se llega a lo que se quiere" (Hombre, 17 años).

Las modificaciones en el papel de proveedor de los hombres como jefes de familia como también el de su paternidad afectiva va permeando generación tras generación, sumado al contexto global que exige y proyecta mayor participación laboral femenina y responsabilidad paternal directa en el cuidado de los/as hijos/as.

Por lo tanto, es en la juventud, donde la igualdad se presenta como un discurso más aceptado y comprendido en torno a las decisiones individuales, y no solo a las necesidades económicas.

\footnotetext{
"Yo no le exigiría que trabajara, pero si ella quiere trabajar... Pero a ella no le daría el derecho que me exigiera" (Hombre, 17 años).
}

Para los jóvenes, la mujer, a pesar de participar en la parte económica del hogar, permanece en sus labores domésticas, como parte de sus obligaciones de género, incrementando la sobrecarga de trabajo y el aumento de las funciones y responsabilidades para suplir las necesidades de los miembros del hogar. Esto las sitúa en una situación, frente a la mirada de los hombres, de sacrificio, pero de crecimiento social, percibiendo la importancia de salir al espacio público y enfrentarlo, así como una reflexión que permite poner el foco en la decisión y la posibilidad de cambiar sobre las personas antes que el género.

"Es que yo pienso que es lo mismo. No creo que sea más fácil ser hombre. Yo pienso que depende de la persona, porque si una mujer quiere trabajar en un 
trabajo de hombre, lo puede hacer también, porque depende de ella" (Hombre, 17 años).

Este tipo de comentarios tiende a contraponerse con lo que se piensa del trabajo doméstico, y con la visión de la importancia del trabajo para los hombres, que ellos mismos comentan. Esto es porque, si bien ellos establecen criterios de igualdad de derechos, tiende a establecerse una valoración de ocupación y dificultad distinta a lo que se puede o no se puede hacer.

Por otro lado, se consolida un nuevo proyecto de futuro en los jóvenes, que considera la idea de formar familia y tener una menor cantidad de hijos, tener una carrera profesional o técnica, colaborar con la familia desde su situación económica alta, y tener una estabilidad socioeconómica antes de tener descendencia, para que su propia familia tenga una buena situación social.

\footnotetext{
"Antes la gente decía "ay, voy a ir a trabajar y voy a tener 20 hijos". Por mí que tenga dos y listo, para andar trayéndolos bien vestidos. Me gustaría tener hijos después de que salga con una carrera, aunque yo estoy seguro que no voy a tener después de mi carrera. Pero, aunque tenga hijos igual voy a terminar mi carrera" (Hombre, 17 años).

"Para tener hijos hay que ser capaz de abastecerse uno primero, porque es una gran responsabilidad, no es un chiste tener hijos de los 20 a los 30 , no es mi idea por lo menos. Tener una casa, algo donde caerme muerto" (Hombre, 17 años).
}

Esto nos habla también de una conciencia de las oportunidades actuales y de un nuevo tipo de responsabilidad frente al costo económico de la crianza que son tomados en cuenta, sobre todo por la experiencia vivida con familiares cercanos. Esto no quiere decir que exista una tendencia a la abstinencia sexual, pues se opina que en las prácticas reales de los jóvenes, no es una opción la negación:

"Pero es que todas dicen eso y después, no todo es posible. Porque no creo que, si en una disco te gusta un niño y quieres acostarte con él, no decís "ay no, no" $y$ te vai de la disco. $Y$ si sale. $Y$ si estay pololeando y tu pololo te pide acostarte, ¿vas a decirle que no?" (Hombre, 18 años).

\section{Análisis sobre la identidad, juventud género y trabajo en sectores rurales}

En el complejo fenómeno de la inserción de los jóvenes en la sociedad adulta, la transición al trabajo deviene como factor central y definitorio del concepto juventud. El estadio adulto se hace sinónimo de integración social y, por lo tanto, la juventud es definida como un proceso de incorporación a la sociedad, ya que implícito al concepto de adulto está el pleno reconocimiento social (Serrano, 1995). Ellos pasan, en un modo clásico de transición, a través de la labor productiva y de ejercer roles de adultos, a un estado de independencia (salario) y por ende, de adultez. De esta forma, es posible afirmar que el trabajo sigue siendo elemento central para la construcción de la identidad (no solo, pero fundamentalmente) de los jóvenes (Agulló, 1997).

No obstante, esto tiende a ser una afirmación certera en la línea identitaria de los jóvenes varones, los que en su conformación de identidad de género -y en este caso, su identidad rural-, se establece a partir y sobre la actividad laboral. Esto es porque, desde los estudios de masculinidades, se plantea que en la construcción de la identidad masculina, es el trabajo (ser proveedor, deberse al trabajo o trabajar por dinero) uno de los atributos que 
distinguen a los varones y forman parte de su identidad, así como el lograr desempeñar ciertos "roles" en cada etapa de su ciclo de vida, expresados por la masculinidad dominante, que es su referente (Olavarría, 2001).

Entendiendo el papel que juega el trabajo como fundamento y núcleo de la identidad y sociabilidad, que explica la profunda legitimidad pública que se deriva de la integración laboral del joven (Serrano, 1995:177), cabe mencionar cómo la transformación de la situación económica desde las décadas 60 y 70, con las modificaciones estructurales del mercado del trabajo, ha generado un contexto especialmente difícil para la población juvenil, puesto que no asegura un espacio de socialización estable, seguro o deseable (desempleo, precariedad y dualización), donde se expone la dependencia y por lo tanto, no se alcanza el estado óptimo de la adultez (Agulló, 1997). El actual modelo de transición (o itinerarios laborales) se caracteriza por la informalización, la segmentación, la intermitencia y la flexibilidad en el ámbito de trabajo, o dicho de otro modo, de precariedad laboral, y enmarca la compleja y diversa situación sociolaboral de los jóvenes (lbíd.). Sin embargo, se ha constatado que el hábitat urbano resulta más obstaculizador que el hábitat rural para el desempeño de una actividad laboral, y por tanto, la consecución de una identidad consolidada presenta mayores dificultades en espacios urbanos (Agulló, 1998). Si bien esto puede haber afectado a gran parte de los jóvenes, según lo observado en nuestra investigación, en el entorno rural el trabajo aún significa una transición a la sociedad adulta, aunque no cumpla con los parámetros deseados, pues el solo hecho de ejercer esta actividad, les entrega un nuevo estatus social, lo cual se complejiza en la idea en que dejan de ser niños, se conso- lidan como hombres, pero también se identifican como jóvenes.

\section{Conclusiones}

Las identidades y relaciones de género se van adecuando y adaptando a los cambios y devenires de la sociedad chilena. Es así como en el transcurso de nuestra historia social podemos apreciar las variaciones contextuales que responden al avance y a las apertura del sector rural a un mundo más globalizado y con procesos de modernización que afectan las comunicaciones, la educación, la información, el desplazamiento y las relaciones de trabajo, además de los cambios políticos que han condicionado la identidad campesina.

En este artículo, vemos a una generación de jóvenes rurales que tienen una temprana conexión con lo urbano, no solo por el traslado que éstos hacen, desde la educación media, hacia sus internados o liceos, sino también por el aumento y la gran presencia de nuevas tecnologías comunicacionales que los vinculan con sujetos y contextos extrarurales, lo que ha abierto una brecha generacional respecto a sus antecesores, sumado al cuestionamiento de la autoridad de los padres y de las escuelas. Además, los jóvenes, en la actualidad, viven en un contexto educacional, diferente al de sus padres y abuelos, y por lo tanto, su educación media completa y sus aspiraciones a la universidad, son entendidas y valoradas como un mecanismo de movilidad social, y los proyecta a nuevas carreras y nuevos desafíos y aspiraciones, modificando proyectos de vida, sus expectativas de futuro y la de sus padres, estableciéndose un mecanismo de quiebre de estatus con sus familias y antepa- 
sados (Vásquez \& Vallejos, 2014). No obstante, también observamos la necesidad y/o responsabilidad que los jóvenes asumen de mantener algunos rasgos de su identidad rural, los lleva a conservar o resignificar ciertos elementos, en su nuevo desarrollo identitario juvenil.

Por otro lado, se muestra también un eje centrado en el trabajo que, si bien, dejó de ser una relación directa con la tierra, conserva los conocimientos y experiencias, mantenidas por generaciones, y sostenidas desde la masculinidad como rito de consolidación de género. Frente a esto, surge como tensión el hecho de que los jóvenes, si bien acceden a fuentes de empleo a temprana edad a través de contactos familiares, entienden las experiencias laborales rurales, especialmente relacionadas a sus padres y abuelos, como sacrificadas y de menor valor, lo que va mermando en su arraigo identitario. En este sentido, es importante mencionar que si bien los trabajos rurales, no son una opción ante sus expectativas de vidas, esto no implica que en su socialización, éstas no sean realizadas y valoradas.

Creemos importante además, reflexionar la forma en que estos jóvenes, a pesar de ser un grupo que expresa de manera más abierta las posibilidades de cambio de roles y de una igualdad de género más efectiva, son quienes cumplen la función de reproductores de ideas y comentarios sexistas, propios del sistema patriarcal tradicional, hacendal. Esto puede reflejar un ámbito de lenta transformación, que caracteriza no solo al ámbito rural -aunque en estos espacios se ve con fuerza- sino que es una tendencia que vemos en nuestra sociedad. A pesar de esto, es fundamental destacar cómo, respecto a las relaciones de género, si bien exhiben ideas sobre la equidad, diversidad, la violencia, suponiendo la importancia de ciertos cambios, los resultados no dan cuenta de transformaciones significativas, y por el contrario, se evidencia la persistencia de ideas tradicionales en torno a lo femenino y a su sexualidad, y al trabajo y rol de proveedor como definición identitaria de los varones.

Como vemos, los testimonios de los jóvenes entrevistados se inscriben en el escenario de una comunidad rural imbuida en un proceso acelerado de hibridación cultural, la cual provoca "respuestas eclécticas en cuanto a la producción y adscripción identitaria juvenil, tensionadas fuertemente por los bienes simbólicos urbanos y las contracciones estructurales propias de la ruralidad" (González, 2003: 172). De esta manera, las nuevas generaciones aportan con interpretaciones del presente y del pasado, pero también son ellas mismas un sujeto en constante transformación, combinando la idealización de la relación hombretierra, y la conservación de elementos identitarios, con la introducción de nuevos códigos juveniles, modas, intereses urbanos, conformándose como un grupo propiamente tal, así como, contraponiendo los avances en relación a los valores en el género, van reinterpretando y reproduciendo las configuraciones tradicionales de su masculinidad y su visión frente a lo femenino y lo masculino.

De esta forma, planteamos que esta red compleja de adscripciones nos entrega herramientas necesarias para abrir una discusión sobre el binarismo que se ha establecido tradicionalmente entre lo urbano y lo rural, y comencemos a ver cómo es posible integrar y resignificar ambos espacios, así como también reflexionar sobre este nuevo status similar a la adultez de un hombre, pero se adscribe a 
una identificación de joven, que transforma ciertas visiones de género, pero que reproduce con fuerza otras. Creemos que este tipo de casos, abren un mundo nuevo y complejo, sobre el futuro de las localidades rurales, sus identidades, y la conformación de un nuevo sujeto joven-varon-rurbano, cuestiones fundamentales para el entendimiento de los actores de nuestro contexto y desarrollo de futuras políticas públicas.

\section{Notas}

1 También existían la masculinidad "libre" en la figura de los gañanes y los peones (Salazar, 1989; Bauer, 1994) cuyo roce era menor, más acotado y con mayores posibilidades de establecer otro tipo de relaciones menos patriarcales ni violentas.

2 Ver por ejemplo, Vio Grossi (1986), Méndez (1986), o en Kmaid (1988) citados en González, 2003.

3 Dentro de los procesos más importantes en la educación que han ocurrido en el periodo de tiempo abarcado en la investigación, es posible mencionar la amplitud de la educación básica, que en seis años se pasó a los 8 años, y posteriormente la reforma a la educación media obligatoria. Además del aporte entregado por el proceso de instalación de internados en ciudades de tamaño intermedio. También se considera el incremento sostenido de la matrícula en la educación superior, ya sea en universidades como en centros de formación técnica e institutos a partir de los años 90 (Rebolledo, 2013).

4 Plan de Desarrollo Comunal PLADECO de la I. Municipalidad de Pumanque 2014-2017, provincia de Colchagua, VI Región de la

\section{Referencias bibliográficas}

Agulló, E. (1997). Jóvenes, trabajo e identidad. Servicio de Publicaciones. Universidad de Oviedo.

(1998). "La centralidad del trabajo en el proceso de construcción de la identidad de los jóvenes: una aproximación psicosocial". Psicothema. № 1.

Astorga, F. (2000). "El canto a lo poeta". Revista musical chilena [online]. №194. Fecha de Consulta: 27 enero 2017]. Disponible en: http://www.scielo.cl/scielo.php?script=sci_arttext\&pid=S071627902000019400007\&lng=es\&nrm=iso

Badinter, E. (1993). "XY. La Identidad Masculina. Ed. Cast.: Alianza Editorial, S.A. Madrid, 1993.
Por último, considerando que el presente trabajo aborda la juventud masculina en una zona agrícola, sería interesante observar cómo los discursos y construcción identitaria se expresa en zonas ganaderas, sus diferencias a lo largo de nuestro país, y cuál es el proceso que viven las mujeres jóvenes rurales, estableciendo líneas de conprension analiticas y comparativas.
Región del Libertador General Bernardo O'Higgins, Chile.

5 Parte de lo que se conoce como "El canto a lo poeta", que es una de las tradiciones más antiguas de Chile y aún se encuentra presente en nuestra zona central. Es la poesía cantada que utiliza principalmente los metros poéticos de la cuarteta o copla y la décima espinela. Se divide en dos grandes grupos: canto a lo divino y canto a lo humano. El primero abarca los temas o fundamentos bíblicos o de carácter religioso, por ejemplo: la creación del mundo, el nacimiento de Cristo, la Virgen María, los Santos, etc. Mientras que el segundo incluye los temas o fundamentos que no son bíblicos ni de carácter religioso, por ejemplo: ponderaciones, literatura, historia humana, amor, contrapuntos, etc. (Astorga, 2000).

6 La denominación "pelás" o "peladas" hacen referencia, en jerga juvenil, a las mujeres cuyas costumbres sexuales las deducen o son conocidas como activas, vivas y con hombres diferentes, relacionándolo con la prostitución y su costumbre de depilarse la entrepierna.
Barrera, I. \& Gormaz, A. (2009). "Adolescentes escolares de la Comuna de Cabrero, Región del Bío-Bío, Chile: fragmentos de una identidad rural en transición”. Antíteses, №3. Disponible en: http://www.uel.br/revistas/uel/index.php/antiteses Fecha consulta: 8 de marzo 2017

Bengoa, J. (1988). Historia social de la agricultura chilena. Tomo I "El poder y la subordinación”. Santiago: Ediciones Sur. (1990). Haciendas y Campesinos. Historia Social de la Agricultura Chilena, Tomo II. Santiago: Ediciones SUR. Colección estudios Históricos.

Duhart, D. (2004). “Juventud Rural en Chile: ¿Problema o 
Solución?’. Última década. №20. Fecha de Consulta: 28 enero 2017. Disponible en: http://www.scielo.cl/scielo.php?script=sci_ arttext\&pid=S0718-22362004000100007\&lng=es\&nrm=iso

Durston, J. (1998). Juventud rural en Brasil y México: reduciendo la invisibilidad. XX Congreso de la Asociación Latinoamericana de Sociología - ALAS. agosto-septiembre, Sao Paulo. Disponible en: http://www.cepal.org/publicaciones/xml/6/4646/indice.html Fecha de consulta: 03 de mayo 2017.

Feixa, C. (1998). El Reloj de Arena. Culturas Juveniles en México. México D.F.: Causa Joven, Colección Jóvenes №4, Instituto Mexicano de la Juventud.

(1999). De jóvenes, bandas y tribus. Antropología de la juventud. Barcelona: Editorial Ariel, S.A.

Fuller, N. (2001). Masculinidad cambios y permanencias. Lima: Pontificia Universidad Católica de Perú Fondo Editorial.

González, Y. (2003). "Juventud rural: Trayectorias teóricas y dilemas identitarios”. Nueva Antropología, №63. Disponible en: http://www.scielo.org.mx/scielo.php?script=sci_arttext\&pid=S0185$06362003000200008 \& \operatorname{lng}=e s \&$ tlng=es Fecha de consulta: 03 de mayo, 2017.

Gómez, S. (1990). "Cambios en la cultura campesina 1965-1990. (Algunas notas)". FLACSO-Chile, Serie Estudios Sociales, № 4.

I. Municipalidad de Pumanque. Plan De Desarrollo Comunal De Pumanque 2014-2017. Disponible en: https://drive.google.com/ file/d/0B6jqtF42VDa_S0ZnNmFLUjNXNHM/view Fecha de consulta: 8 de marzo 2017.

Lamas, M. (2002). Cuerpo, Diferencia Sexual y Género. España: Editorial Taurus.

Méndez, L. (1986), "La impertinencia de hablar de juventud rural", CREA, México.

Olavarría, J. (2001). "Invisibilidad y Poder. Varones de Santiago de Chile". En: M. Viveros, J. Olavarría \& N. Fuller. (2001). Hombres e identidades de género: investigaciones desde América Latina. Bogotá: Colección CES. Universidad Nacional de Colombia.

Rebolledo, L. (1997). "Las mujeres rurales en el contexto de la modernización agraria". Revista Anales, № 5. Disponible en: http:// www.revistas.uchile.cl/index.php/ANUC/article/viewFile/2987/2865 (2013). Identidades de género y transformaciones en el mundo rural. La situación en Chile central. Avance de investigación en curso, GT 11, Acta Científica XXIX Congreso de la Asociación Latinoamericana de Sociología 2013. Disponible en: http:// actacientifica.servicioit.cl/biblioteca/gt/GT11/GT11_LoretoRebolledo. pdf Fecha de consulta: 04 de mayo 2017.

Reyes, A. (2009). "La escuela secundaria como espacio de construcción de identidades juveniles". Revista mexicana de investigación educativa, №40.

Rocha, T. (2009). "Desarrollo de la identidad de género desde una perspectiva psico-socio-cultural: Un recorrido Conceptual”. Revista
Interamericana de Psicología, №2.

Rojas, J. (2004). Los trabajadores chilenos desde la colonia hasta 1973. Instituto de Ciencias Alejandro Lipschutz. Disponible en: http://www.bibliotecaobrera.cl/wp-content/uploads/2008/10/lostrabajadores-chilenos-desde-la-colonia-hasta-1973-jorge-rojas-flores. pdf Fecha de Consulta: Julio de 2012

Romero, P. (2006). "Masculinidades Juveniles y Tensiones Identitarias: un enfoque de género". Revista del Programa Andino de Derechos Humanos. №16.

Salazar, G. (1989). Labradores, peones y proletarios (Siglo XIX). [Libro]. Colección Estudios Históricos. Santiago de Chile: Ediciones SUR. Disponible en: http://www.sitiosur.cl/r.php?id=233. Fecha de Consulta: 16 julio 2012.

Serrano, A. (1995). "Procesos paradójicos de construcción de la juventud en un contexto de crisis del mercado de trabajo". Revista Española de Investigaciones Sociológicas. № 71/72. Disponible en: http://www.jstor.org/stable/40183867

Tinsman, H. (2009). La tierra para el que la trabaja: Género, sexualidad y movimientos campesinos en la reforma Agraria Chilena. Santiago: Editorial LOM.

Valdés, X., Rebolledo, L. \& Willson, A. (1995). Masculino y Femenino en la hacienda del siglo XX. Santiago: FONDART - CEDEM.

Valdés, T., \& Olavarría, J. (eds.), (1997). Masculinidad/es. Poder y Crisis. Santiago: Ediciones de las Mujeres № 24, ISIS Internacional, FLACSO-Chile.

Valdés, X. (1998). "Temporeros y temporeras de la fruta. modernización del agro y cambio en las relaciones sociales de género". Revista Proposiciones, № 28.

(2000). "Masculinidad en el mundo rural: realidades que cambian, símbolos que permanecen". En Masculinidad/es. Identidad, sexualidad y familia. Santiago: Ediciones FLACSO.

Vásquez, C. \& Vallejos, D. (2014). "Migración juvenil rural en la región del Maule, Chile. Expectativas de futuro de la nueva generación”. Revista de Ciencias Sociales, DS-FCS №35. Montevideo.

Vio Grossi (1986): “Juventud rural, ¿nuevos actores en el campo?”; Méndez (1986), "La impertinencia de hablar de juventud rural”. Programa interdisciplinario de la educación PIIE. Santiago de Chile.

Viveros, M. (2000). "Notas en torno a la categoría analítica de género", en: A, Robledo \& Y, Puyana (Comp.) Ética: masculinidades y feminidades, Bogotá: Centro de Estudios Sociales, Universidad Nacional de Colombia.

Viveros, M., Olavarría, J., \& Fuller, N. (2001). Hombres e identidades de género. Investigaciones desde América Latina. Universidad Nacional de Colombia. Facultad de Ciencias Humanas. Centro de Estudios Sociales. Julio.

Zapata, S. (2000). Al encuentro del joven rural. Agencia para la cooperación del IICA en Chile. 